\title{
Winrich Kühne
}

\section{Blauhelme in einer turbulenten Welt}

Beiträge internationaler Experten zur Fortentwicklung des Völkerrechts und der Vereinten Nationen

Nomos Verlagsgesellschaft, Baden-Baden 1993, 571 S., DM 49,--

Bis jetzt hat sich die deutsche Öffentlichkeit hauptsächlich mit dem Problem der verfassungsrechtlichen Zulässigkeit des Einsatzes von Bundeswehrsoldaten beschäftigt, wenn die internationale Friedenssicherung der UNO diskutiert wurde. Das vorliegende Buch geht weit über diesen Rahmen hinaus und will weiterführende Informationen über die Fortentwicklung der traditionellen Blauhelmeinsätze mit ihren militärischen und zivilen Komponenten, die Zusammenarbeit mit regionalen Einrichtungen, Ausbaumöglichkeiten, präventiver Diplomatie, Konfliktverhütung, Finanzierungsprobleme, sowie die Fortentwicklung des Völkerrechts und der UNO-Charta vermitteln.

"Das Eingreifen der VN kommt fast immer zu spät, das heißt zu einem Zeitpunkt, an dem die Konflikte eine Eigendynamik entwickelt haben, die durch externe Akteure entweder gar nicht oder nur durch den Einsatz massiver Mittel zu beeinflussen ist" (S. 71). Als Beispiel dafür wird der Krieg in Jugoslawien angeführt, vor dem die CIA bereits im Herbst 1990 deutlich gewarnt hatte. Ein effektives internationales System für Sicherheit und Frieden "requires both the creation of conditions in which peace can be maintained, and the capacity to anticipate and to prevent breaches of the peace (...). Governments will also have to be prepared to support and put adequate resources behind both global and regional security systems" ${ }^{1}$.

Für die Hauptentwicklungsaufgabe der Vereinten Nationen in den 90er Jahren hält man das 'robuste peace-keeping', das eine aus den fünf zur Zeit bestehenden Kategorien von VN-Truppen weiterentwickelte Form der Friedenssicherung darstellt.

Über "Wirksameres Völkerrecht oder neues Weltinnenrecht" macht sich Jost Delbrück Gedanken. Er betont die Vergemeinschaftung der Verantwortung. "Es wird in den kommenden Jahren die Aufgabe der Völkerrechtswissenschaft sein, das neue Weltinnenrecht als Rechtsordnung einer globalen Weltgesellschaft näher zu bestimmen und in seinem Verhältnis zu dem lange Zeit gewiß noch weiterbestehenden modernen Völkerrecht dogmatisch zu erfassen" (S. 131).

Nach einer Aufzählung des Instrumentariums für das Krisenmanagement, der sich wandelnden Rolle des Sicherheitsrats und seiner Aufgaben im nächsten Jahrzehnt werden die humanitären Einsätze erläutert. Eines der Hauptthemen ist die 'Agenda for Peace'; besondere Aufmerksamkeit ist hier den fünf 'recommendations' von Thomas Weiss zu schenken.

Regionale Friedenssicherung und Beispiele aus Lateinamerika und Südostasien führen zum Problem des russischen peace-keeping in den Staaten der ehemaligen Sowjetunion. "Armed conflict is becoming a dominant feature of the landscape in the former Soviet

Urquhart, Leaming from the Gulf, in: The New York Times Review of Books, 7.3.1991, p. 34. 
Union (...). Moscow has placed particular emphasis on the idea of sending peace-keeping troops to conflict areas (...). Considering strong indications that Russia's military may be playing the role of co-belligerent in some areas, Russia's bona fides as a potential peacekeeper should be questioned" (S. 351). Zwei Grundsätze des peace-keeping wurden in Fällen wie Moldawien oder Nagorni-Karabach ignoriert: Erstens schickte man 'Friedenstruppen' in Gebiete, in denen der Konflikt schon / noch voll entbrannt war, und zweitens waren diese Truppen auch nicht neutral. "Some in Russia argue that peace-keeping should be undertaken when Russia's security is or could be threatened" (S. 368), eine Philosophie, die ein interessantes Licht auf die russischen Friedenstruppen im ehemaligen Jugoslawien wirft.

Es folgen Überlegungen zu friedlicher Streitbeilegung, Verbesserung des Systems allgemein, Gedanken zur Kasuistik internationaler Interventionen (gibt es eine Einmischungspflicht?). 'Höhepunkte' sind zweifellos auch die beiden Aufsätze von Pia Bungarten und Hartwig Hummel zu "U.S. Policies" und "Japanischen Blauhelmeinsätzen". "Going out Alone, but in Multilateral Company", the willingness to assume the responsibilities of leadership, "sharing costs? - sharing power?" (S. 452) - Ansichten der Amerikaner. Im Detail werden die Schritte zu einer neuen Verfassungsinterpretation in Japan und das Gesetz über Blauhelmeinsätze analysiert - Vorbildfunktion der Japaner?

Trotz winziger Abstriche (den Namen eines so bekannten Kollegen wie Thomas M. Franck darf man einfach nicht wiederholt falsch schreiben!) ist das Buch wirklich gelungen. Es ist gut zu lesen, sof ern man die deutsche wie die englische Sprache gleichermaßen gut beherrscht (mehr als die Hälfte der Beiträge sind in englischer Sprache verfaßt), was wohl eines der Indizien dafür ist, daß die Sammlung doch eher für Fachleute als für die breite Öffentlichkeit geschrieben ist. Deutlich umfangreicher an Themen wie Details als vergleichbare Arbeiten bringt es einige ganz neue Aspekte und verbindet 'geschickt' Blauhelmeinsätze in Geschichte und Gegenwart mit Zukunftsperspektiven, macht klar, welche Voraussetzungen geschaffen wurden oder noch geschaffen werden müssen, gibt Beispiele für Erfolge und Mißerfolge und zeigt Wege auf, die andere Länder zum peace-keeping gegangen sind. Das Beispiel Japans sollten wir Deutsche besonders aufmerksam lesen! Die gelungene Synthese von Völkerrecht und Politik verdankt das Buch vielleicht auch der Tatsache, daß so viele international namhafte Experten daran mitgewirkt haben. 